\title{
Circular plant peptides as templates for GPCR drug discovery?
}

\author{
Johannes Koehbach', Margaret O'Brien², Marion Miazzo ${ }^{1}$, David J Craik², Michael Freissmuth', Christian W Gruber ${ }^{\text {* }}$ \\ From 17th Scientific Symposium of the Austrian Pharmacological Society (APHAR). Joint meeting with the \\ Hungarian Society of Experimental and Clinical Pharmacology (MFT) \\ Innsbruck, Austria. 29-30 September 2011
}

\section{Background}

Cyclotides are disulfide-rich miniproteins with the unique structural features of a circular backbone and knotted arrangement of three conserved disulfide bonds. These features make them exceptionally stable and they have applications as plant defense (insecticidal) agents and stable drug frameworks. So far they have been found mainly in two plant families, including in every species of the violet family (Violaceae) so far examined, and in a few species of the coffee family (Rubiaceae).

\section{Methods}

We optimized the cyclotide screening protocol using MALDI-TOF/TOF and two dimensional nano LC-MS/ MS followed by manual and automated peptide sequence assignments. Biological analysis was carried out by collagen uterine contractility assays and pharmacological data were generated by competitive displacement binding and functional GPCR luciferase assays.

\section{Results}

Using the rapid cyclotide discovery workflow, we analyzed $>300$ flowering plant species and confirmed the presence of cyclotides in several plant families. On the basis of the phylogeny of cyclotide-bearing plants and the analysis of precursor gene sequences, we have refined the current hypothesis of the evolution of circular plant peptides. Based on the traditional usage of the plant Oldenlandia affinis as an uterotonic agent, we have tested and identified cyclotides with oxytocin-like activity and identified the oxytocin receptor, as representative member of the GPCR family, as a molecular target of cyclotides.

\section{Conclusions}

Cyclotides are interesting targets for pharmaceutical applications due to their unique structural framework, their bioactivities and sequence diversity. Based on their predicted number of tens of thousands, which potentially makes them one of the largest protein families within the plant kingdom, they constitute an immense combinatorial library of natural peptides, which is accessible for drug discovery.

\section{Acknowledgements}

This work is funded by the Austrian Science Fund FWF (P22889).

\section{Author details}

${ }^{1}$ Institute of Pharmacology, Center of Physiology and Pharmacology, Medical University of Vienna, 1090 Vienna, Austria. ${ }^{2}$ National Center for Biomedical and Engineering Science, National University of Ireland Galway, Galway, Ireland. ${ }^{3}$ Institute for Molecular Biosciences, University of Queensland, St. Lucia, QLD 4072, Australia.

Published: 5 September 2011

doi:10.1186/1471-2210-11-S2-A16

Cite this article as: Koehbach et al:: Circular plant peptides as templates for GPCR drug discovery? BMC Pharmacology 2011 11(Suppl 2):A16.

\footnotetext{
* Correspondence: christian.w.gruber@meduniwien.ac.at

${ }^{1}$ Institute of Pharmacology, Center of Physiology and Pharmacology, Medical University of Vienna, 1090 Vienna, Austria

Full list of author information is available at the end of the article
} 